\title{
THE STRICT TOPOLOGY ON THE DISCRETE LEBESGUE SPACES
}

\author{
SAEID MAGHSOUDI ${ }^{\circledR}$ and RASOUL NASR-ISFAHANI
}

(Received 10 May 2010)

\begin{abstract}
Let $\Sigma$ be a set and $\sigma$ be a positive function on $\Sigma$. We introduce and study a locally convex topology $\beta^{1}(\Sigma, \sigma)$ on the space $\ell^{1}(\Sigma, \sigma)$ such that the strong dual of $\left(\ell^{1}(\Sigma, \sigma), \beta^{1}(\Sigma, \sigma)\right)$ can be identified with the Banach space $\left(c_{0}(\Sigma, 1 / \sigma),\|\cdot\|_{\infty, \sigma}\right)$. We also show that, except for the case where $\Sigma$ is finite, there are infinitely many such locally convex topologies on $\ell^{1}(\Sigma, \sigma)$. Finally, we investigate some other properties of the locally convex space $\left(\ell^{1}(\Sigma, \sigma), \beta^{1}(\Sigma, \sigma)\right)$, and as an application, we answer partially a question raised by A. I. Singh [ ${ }^{\circ} L_{0}^{\infty}(G)^{*}$ as the second dual of the group algebra $L^{1}(G)$ with a locally convex topology', Michigan Math. J. 46 (1999), 143-150].
\end{abstract}

2010 Mathematics subject classification: primary 46A03; secondary 46E30, 46H05.

Keywords and phrases: discrete Lebesgue space, locally convex space, strict topology, strong dual.

\section{Introduction}

Throughout this paper, let $\Sigma$ be an arbitrary set and $\sigma$ be a positive function on $\Sigma$. We denote by $\ell^{1}(\Sigma, \sigma)$ the space of all complex-valued functions $\varphi$ on $\Sigma$ such that $\sigma \varphi \in \ell^{1}(\Sigma)$, the usual Lebesgue space of the discrete space $\Sigma$. Then $\ell^{1}(\Sigma, \sigma)$ with the norm $\|\cdot\|_{1, \sigma}$ defined by

$$
\|\varphi\|_{1, \sigma}:=\|\sigma \varphi\|_{1}
$$

is a Banach space. For each $x \in \Sigma$, we denote by $\delta_{x}$ the function defined on $\Sigma$ by $\delta_{x}(t)=1$ for $t=x$ and $\delta_{x}(t)=0$ otherwise. Also, let $\ell^{\infty}(\Sigma, 1 / \sigma)$ denote the space of all complex-valued functions $f$ on $\Sigma$ with $f / \sigma \in \ell^{\infty}(\Sigma)$, the space of all bounded functions on $\Sigma$. Then $\ell^{\infty}(\Sigma, 1 / \sigma)$ with the norm $\|\cdot\|_{\infty, \sigma}$ defined by

$$
\|f\|_{\infty, \sigma}=\|f / \sigma\|_{\infty}
$$

is a Banach space. Moreover, $\ell^{\infty}(\Sigma, 1 / \sigma)$ is the dual of $\ell^{1}(\Sigma, \sigma)$ by the pairing

$$
\langle\theta(f), \varphi\rangle:=\sum_{x \in \Sigma} f(x) \varphi(x) \quad\left(f \in \ell^{\infty}(\Sigma, 1 / \sigma), \varphi \in \ell^{1}(\Sigma, \sigma)\right) .
$$

(C) 2010 Australian Mathematical Publishing Association Inc. 0004-9727/2010 \$16.00 
Denote by $c_{0}(\Sigma, 1 / \sigma)$ the subspace of $\ell^{\infty}(\Sigma, 1 / \sigma)$ consisting of all functions $f$ on $\Sigma$ with $f / \sigma \in c_{0}(\Sigma)$, the space of all functions on $\Sigma$ vanishing at infinity, and note that $\ell^{1}(\Sigma, \sigma)$ is the dual of $c_{0}(\Sigma, 1 / \sigma)$ under the above duality.

The study of the strict topology on $C(X)$, the space of continuous functions on the topological space $X$, began with Buck's work in [1]. There is an extensive literature on this subject; see, for example, [10, 11]. Also, for such a study in another context, see $[4,14,16]$, for example. For a generalization of the strict topology and/or strict topology in a more general setting, see, for example, [2, 7].

In this paper, we introduce and study a locally convex topology $\beta^{1}(\Sigma, \sigma)$ on $\ell^{1}(\Sigma, \sigma)$ such that $c_{0}(\Sigma, 1 / \sigma)$ can be identified with the strong dual of $\ell^{1}(\Sigma, \sigma)$. We then show that, except for the trivial case where $\Sigma$ is finite, there are infinitely many such locally convex topologies $\tau$ on $\ell^{1}(\Sigma, \sigma)$, and hence $c_{0}(\Sigma, 1 / \sigma)$ can be considered as the strong dual of $\ell^{1}(\Sigma, \sigma)$. We study, among other things, some locally convex space properties of the space $\left(\ell^{1}(\Sigma, \sigma), \beta^{1}(\Sigma, \sigma)\right)$. Finally, we give a partial answer to a question raised by Singh in [12].

\section{A locally convex topology on $\ell^{1}(\Sigma, \sigma)$}

Let $\Sigma$ be a set and $\sigma: \Sigma \longrightarrow(0, \infty)$. The set of increasing sequences of finite subsets of $\Sigma$ is denoted by $\mathcal{F}$ and the set of increasing sequences $\left(r_{n}\right)$ of real numbers in $(0, \infty)$ with $r_{n} \rightarrow \infty$ by $\mathcal{R}$. For any $\left(F_{n}\right) \in \mathcal{F}$ and $\left(r_{n}\right) \in \mathcal{R}$, set

$$
U\left(\left(F_{n}\right),\left(r_{n}\right)\right):=\left\{\varphi \in \ell^{1}(\Sigma, \sigma): \sum_{x \in F_{n}}|\varphi(x)| \sigma(x) \leq r_{n} \text { for all } n \geq 1\right\},
$$

and note that $U\left(\left(F_{n}\right),\left(r_{n}\right)\right)$ is a convex balanced absorbing set in the space $\ell^{1}(\Sigma, \sigma)$. It is easy to see that the family $\mathcal{U}$ of all sets $U\left(\left(F_{n}\right),\left(r_{n}\right)\right)$, for $\left(F_{n}\right) \in \mathcal{F}$ and $\left(r_{n}\right) \in \mathcal{R}$, is a base of neighbourhoods of zero for a locally convex topology on $\ell^{1}(\Sigma, \sigma)$; see, for example, [13, Theorem 1.18]. We denote this topology by $\beta^{1}(\Sigma, \sigma)$ and call it the strict topology on $\ell^{1}(\Sigma, \sigma)$. Note that the strict topology can be generated by the family $\left\{\mathcal{P}_{U}: U \in \mathcal{U}\right\}$ of seminorms on $\ell^{1}(\Sigma, \sigma)$, where

$$
\mathcal{P}_{U}(\varphi):=\sup \left\{r_{n}^{-1} \sum_{x \in F_{n}}|\varphi(x)| \sigma(x): n \geq 1\right\}
$$

for all $\varphi \in \ell^{1}(\Sigma, \sigma)$ and $U:=U\left(\left(F_{n}\right),\left(r_{n}\right)\right) \in \mathcal{U}$. We denote the norm topology on $\ell^{1}(\Sigma, \sigma)$ by $n(\Sigma, \sigma)$; note that $\beta^{1}(\Sigma, \sigma) \leq n(\Sigma, \sigma)$.

Proposition 2.1. Let $\Sigma$ be an infinite set and $\sigma$ be a positive function on $\Sigma$. Then a subset of $\ell^{1}(\Sigma, \sigma)$ is $n(\Sigma, \sigma)$-bounded if and only if it is $\beta^{1}(\Sigma, \sigma)$-bounded.

Proof. Let $B$ be a $\beta^{1}(\Sigma, \sigma)$-bounded set in $\ell^{1}(\Sigma, \sigma)$, and suppose that $B$ is not $n(\Sigma, \sigma)$-bounded. Then there is a sequence $\left(\varphi_{n}\right) \subseteq B$ such that $\left\|\varphi_{n}\right\|_{1, \sigma}>n$ for all $n \geq 1$. For each $n \geq 1$, choose a finite set $F_{n}$ in $\Sigma$ such that

$$
\sum_{x \in F_{n}}\left|\varphi_{n}(x)\right| \sigma(x) \geq n
$$


and note that $\left(F_{n}\right) \in \mathcal{F}$. Let $\left(r_{n}\right)$ be a sequence in $\mathcal{R}$ with $r_{n}^{2} \geq n$. Since $B$ is $\beta^{1}(\Sigma, \sigma)$ bounded, there is a constant $s>0$ such that

$$
B \subseteq s U\left(\left(F_{n}\right),\left(r_{n}\right)\right)
$$

for all $n \geq 1$. We therefore have

$$
n \leq \sum_{x \in F_{n}}\left|\varphi_{n}(x)\right| \sigma(x)<r_{n} s
$$

which is a contradiction. The converse is clear.

We denote by $\tau_{b}(\Sigma, \sigma)$ the strong topology on $\left(\ell^{1}(\Sigma, \sigma), \beta^{1}(\Sigma, \sigma)\right)^{*}$; that is, the topology of uniform convergence on bounded subsets of $\ell^{1}(\Sigma, \sigma)$ with respect to the weak topology $\sigma\left(\ell^{1}(\Sigma, \sigma),\left(\ell^{1}(\Sigma, \sigma), \beta^{1}(\Sigma, \sigma)\right)^{*}\right)$. We also denote the topology given on $\left(\ell^{1}(\Sigma, \sigma), \beta^{1}(\Sigma, \sigma)\right)^{*}$ by the norm

$$
\|f\|=\sup \left\{|f(\varphi)|: \varphi \in \ell^{1}(\Sigma, \sigma),\|\varphi\|_{1, \sigma}=1\right\},
$$

by $\tau_{n}(\Sigma, \sigma)$. An immediate consequence of Proposition 2.1 is that on $\left(\ell^{1}(\Sigma, \sigma)\right.$, $\left.\beta^{1}(\Sigma, \sigma)\right)^{*}$ the strong topology $\tau_{b}(\Sigma, \sigma)$ coincides with the topology $\tau_{n}(\Sigma, \sigma)$.

PROPOSITION 2.2. Let $\Sigma$ be a set and $\sigma$ be a positive function on $\Sigma$. On $\ell^{1}(\Sigma, \sigma)$ the norm topology $n(\Sigma, \sigma)$ coincides with the strict topology $\beta^{1}(\Sigma, \sigma)$ if and only if $\Sigma$ is finite.

Proof. Consider the set

$$
U:=\left\{\varphi \in \ell^{1}(\Sigma, \sigma):\|\varphi\|_{1, \sigma}<1\right\}
$$

and note that $U$ is $n(\Sigma, \sigma)$-open, and thus $\beta^{1}(\Sigma, \sigma)$-open. It follows that there is a sequence $\left(\left(F_{n}\right),\left(r_{n}\right)\right)$ in $\mathcal{F} \times \mathcal{R}$ such that $U\left(\left(F_{n}\right),\left(r_{n}\right)\right) \in U$. Suppose that $\Sigma$ is not finite, so we can choose $n_{0}$ such that $r_{n_{0}}>1$ and $x_{n_{0}} \in \Sigma \backslash F_{n_{0}}$. Let

$$
\varphi:=\sigma\left(x_{n_{0}}\right)^{-1} \delta_{x_{n_{0}}} .
$$

We then have $\varphi \in U\left(\left(F_{n}\right),\left(r_{n}\right)\right)$, but $\varphi \notin U$.

\section{Dual of $\ell^{1}(\Sigma, \sigma)$ with the strict topology}

We commence this section with the following key result.

THEOREM 3.1. Let $\Sigma$ be a set and $\sigma$ be a positive function on $\Sigma$. Let $\tau$ be a locally convex topology on $\ell^{1}(\Sigma, \sigma)$ with $\sigma_{0}(\Sigma, \sigma) \leq \tau \leq \beta^{1}(\Sigma, \sigma)$. Then the dual of $\left(\ell^{1}(\Sigma, \sigma), \tau\right)$ endowed with the strong topology can be identified with $c_{0}(\Sigma, 1 / \sigma)$ endowed with $\|\cdot\|_{\infty, \sigma}$-topology.

PROOF. It is sufficient to prove the theorem for the case $\tau=\beta^{1}(\Sigma, \sigma)$. To this end we first show that

$$
\theta\left(c_{0}(\Sigma, 1 / \sigma)\right) \subseteq\left(\ell^{1}(\Sigma, \sigma), \beta^{1}(\Sigma, \sigma)\right)^{*}
$$


Let $f$ be in $c_{0}(\Sigma, 1 / \sigma)$ and $\varepsilon>0$ be given. Choose an element $\left(\left(F_{n}\right),\left(r_{n}\right)\right)$ of $\mathcal{F} \times \mathcal{R}$ with $r_{n} \longrightarrow \infty$ and $r_{1} \geq 2$ such that

$$
|f(x)| \leq \varepsilon r_{n}^{-2} \sigma(x) \quad(n \geq 1)
$$

for $x \in \Sigma \backslash F_{n}$. We show that

$$
|\langle\theta(f), \varphi\rangle| \leq \varepsilon \quad \text { for all } \varphi \in U\left(\left(F_{n}\right),\left(r_{n}\right)\right)
$$

from which it follows that $\theta(f) \in\left(\ell^{1}(\Sigma, \sigma), \beta^{1}(\Sigma, \sigma)\right)^{*}$.

To this end, let $\varphi \in U\left(\left(F_{n}\right),\left(r_{n}\right)\right)$, and set $F_{0}=\emptyset$ and $r_{0}=2$. Since $f(x)=0$ for all $x \in \Sigma \backslash \bigcup_{n=2}^{\infty} F_{n}$, it follows from

$$
\bigcup_{n=2}^{\infty} F_{n}=\bigcup_{n=0}^{\infty}\left(F_{n+1} \backslash F_{n}\right)
$$

that

$$
\begin{aligned}
|\langle\theta(f), \varphi\rangle| & =\left|\sum_{x \in \Sigma} g(x) \varphi(x)\right| \\
& \leq \sum_{x \in \bigcup_{n=2}^{\infty} F_{n}}|f(x) \| \varphi(x)| \\
& \leq \sum_{n=0}^{\infty}\left(\sum_{x \in F_{n+1} \backslash F_{n}}|f(x)||\varphi(x)|\right) \\
& \leq \sum_{n=0}^{\infty} \varepsilon r_{n}^{-2}\left(\sum_{x \in F_{n+1} \backslash F_{n}}|\varphi(x)| \sigma(x)\right) .
\end{aligned}
$$

On the other hand,

$$
\begin{aligned}
\sum_{n=0}^{m} r_{n}^{-2}\left(\sum_{x \in F_{n+1} \backslash F_{n}}|\varphi(x)| \sigma(x)\right)= & \sum_{n=0}^{m}\left(r_{n}^{-2}-r_{n+1}^{-2}\right)\left(\sum_{x \in F_{n+1} \backslash F_{1}}|\varphi(x)| \sigma(x)\right) \\
& +r_{m+1}^{-2} \sum_{x \in F_{m+1} \backslash F_{1}}|\varphi(x)| \sigma(x) \\
\leq & \sum_{n=0}^{m} 2\left(r_{n}^{-1}-r_{n+1}^{-1}\right) r_{n}^{-1}\left(\sum_{x \in F_{n+1}}|\varphi(x)| \sigma(x)\right) \\
& +r_{m+1}^{-2} \sum_{x \in F_{m+1}}|\varphi(x)| \sigma(x) \\
\leq & \sum_{n=0}^{m} 2\left(r_{n}^{-1}-r_{n+1}^{-1}\right)+r_{m+1}^{-1} .
\end{aligned}
$$

Thus,

$$
|\langle\theta(f), \varphi\rangle| \leq \varepsilon\left(2 r_{0}^{-1}-r_{m+1}^{-1}\right)<\varepsilon
$$


This shows that

$$
\theta(f) \in\left(\ell^{1}(\Sigma, \sigma), \beta^{1}(\Sigma, \sigma)\right)^{*} .
$$

Now, let $H$ be a $\beta^{1}(\Sigma, \sigma)$-continuous functional on $\ell^{1}(\Sigma, \sigma)$. Then there is an element $\left(\left(F_{n}\right),\left(r_{n}\right)\right)$ in $\mathcal{F} \times \mathcal{R}$ such that

$$
|\langle H, \varphi\rangle|<1 \quad \text { for all } \varphi \in U\left(\left(F_{n}\right),\left(r_{n}\right)\right) .
$$

It is clear that $H$ is also norm continuous on $\ell^{1}(\Sigma, \sigma)$. It follows that $H=\theta(f)$ for some $f \in \ell^{\infty}(\Sigma, 1 / \sigma)$. We show that $f \in c_{0}(\Sigma, 1 / \sigma)$. It suffices to prove that

$$
|f(x)| \leq \sigma(x) r_{n}^{-1}
$$

for all $n \geq 1$ and all $x \in \Sigma \backslash F_{n}$.

To this end, suppose on the contrary that there exist $m \geq 1$ and $x_{0} \in \Sigma \backslash F_{m}$ such that

$$
\left|f\left(x_{0}\right)\right|>\sigma\left(x_{0}\right) r_{m}^{-1} .
$$

Thus, there is a function $g \in \ell^{\infty}(\Sigma, 1 / \sigma)$ such that $g f=|f| \sigma$ and $\|g\|_{\infty, \sigma} \leq 1$. Let $\varphi$ be a function in $\ell^{1}(\Sigma, \sigma)$ with

$$
\sigma^{2} \varphi=r_{m} g \delta_{x_{0}}
$$

Then

$$
\begin{aligned}
\left|\sum_{x \in \Sigma} f(x) \varphi(x)\right| & =\left|\sum_{x \in \Sigma} \frac{r_{m} g f \delta_{x_{0}}}{\sigma^{2}}\right| \\
& =r_{m} \frac{\left|f\left(x_{0}\right)\right|}{\sigma\left(x_{0}\right)} \\
& >1 .
\end{aligned}
$$

That is, $|\langle H, \varphi\rangle|>1$ which contradicts the fact that $\varphi \in U\left(\left(F_{n}\right),\left(r_{n}\right)\right)$. Therefore,

$$
\theta\left(c_{0}(\Sigma, 1 / \sigma)\right)=\left(\ell^{1}(\Sigma, \sigma), \beta^{1}(\Sigma, \sigma)\right)^{*}
$$

Moreover, $\|f\|_{\infty, \sigma}=\|\theta(f)\|$ for all $f \in c_{0}(\Sigma, 1 / \sigma)$. Now, invoke Proposition 2.1 to conclude that $\theta$ is an identification from $c_{0}(\Sigma, 1 / \sigma)$ endowed with the $\|\cdot\|_{\infty, \sigma^{-}}$ topology onto $\left.\left(\ell^{1}(\Sigma, \sigma)\right), \beta^{1}(\Sigma, \sigma)\right)^{*}$ endowed with the norm topology.

We denote by $\sigma_{0}(\Sigma, \sigma)$ the weak topology $\sigma\left(\ell^{1}(\Sigma, \sigma), \theta\left(c_{0}(\Sigma, 1 / \sigma)\right)\right)$. Let us remark that

$$
\sigma_{0}(\Sigma, \sigma) \leq \beta^{1}(\Sigma, \sigma) \leq n(\Sigma, \sigma)
$$

Proposition 3.2. Let $\Sigma$ be a set and $\sigma$ be a positive function on $\Sigma$. Then the weak topology $\sigma_{0}(\Sigma, \sigma)$ on $\ell^{1}(\Sigma, \sigma)$ coincides with the strict topology $\beta^{1}(\Sigma, \sigma)$ if and only if $\Sigma$ is finite.

PROOF. Suppose that $\Sigma$ is infinite. Let $\left(F_{n}\right) \in \mathcal{F}$ be an increasing sequence with $F_{0}=\emptyset$. So, if $r_{n}=n$, then $U\left(\left(F_{n}\right),\left(r_{n}\right)\right)$ is a $\beta^{1}(\Sigma, \sigma)$-neighbourhood of zero. 
Let $E$ be the subspace of $\ell^{1}(\Sigma, \sigma)$ consisting of all $\varphi \in \ell^{1}(\Sigma, \sigma)$ with

$$
\sum_{x \in F_{n}} \varphi(x) \sigma(x)=0 \quad \text { for all } n \geq 1,
$$

and note that $\varphi_{n} \notin E$, where $\varphi_{n}=\chi_{F_{n} \backslash F_{n-1}}$. Then $E$ has infinite codimension in $\ell^{1}(\Sigma, \sigma)$. It follows that any subspace $F$ of $\ell^{1}(\Sigma, \sigma)$ contained in $U\left(\left(F_{n}\right),\left(r_{n}\right)\right)$ has infinite codimension; this is because $F \subset E$. Since any $\sigma_{0}(\Sigma, \sigma)$-neighbourhood of zero contains a subspace of $\ell^{1}(\Sigma, \sigma)$ with finite codimension, $U\left(\left(F_{n}\right),\left(r_{n}\right)\right)$ is not a $\sigma_{0}(\Sigma, \sigma)$-neighbourhood of zero, whereas it is a $\beta^{1}(\Sigma, \sigma)$-neighbourhood.

Corollary 3.3. Let $\Sigma$ be an infinite set and $\sigma$ be a positive function on $\Sigma$. Then there exist uncountably many locally convex topologies $\tau$ on $\ell^{1}(\Sigma, \sigma)$ such that $\sigma_{0}(\Sigma, \sigma) \leq \tau \leq \beta^{1}(\Sigma, \sigma)$.

Proof. Since $\Sigma$ is infinite, Proposition 3.2 implies that $\sigma_{0}(\Sigma, \sigma)<\beta^{1}(\Sigma, \sigma)$. We now only need to recall from [8] that the only case in which the dual pair generates a finite number of polar topologies is when all polar topologies are equal to the weak topology.

\section{Some properties of the strict topology}

In this section, we investigate the strict topology on $\ell^{1}(\Sigma, \sigma)$ as a locally convex topology.

Proposition 4.1. Let $\Sigma$ be a set and $\sigma$ be a positive function on $\Sigma$. The locally convex space $\left(\ell^{1}(\Sigma, \sigma), \beta^{1}(\Sigma, \sigma)\right)$ is complete.

Proof. Let $\left(\varphi_{\alpha}\right)$ be a $\beta^{1}(\Sigma, \sigma)$-Cauchy net in $\ell^{1}(\Sigma, \sigma)$. Obviously, we can find a function $\varphi$ on $\Sigma$ such that $\left(\varphi_{\alpha}\right)$ converges to $\varphi$ in the pointwise topology. Suppose towards a contradiction that $\varphi$ is not in $\ell^{1}(\Sigma, \sigma)$. Then we can find a sequence $\left(x_{n}\right)$ in $\Sigma$ such that

$$
\sum_{i=1}^{k_{n}}\left|\varphi\left(x_{i}\right)\right| \sigma\left(x_{i}\right) \geq 2 k_{n}
$$

for all $n \geq 1$, where $1<k_{1}<k_{2}<\cdots$. Let $F_{n}:=\left\{x_{k_{1}}, x_{k_{2}}, \ldots, x_{k_{n}}\right\}$ and $r_{n}:=k_{n}$. There exists $\alpha_{0}$ such that

$$
\sum_{x \in F_{n}}\left|\varphi_{\alpha}(x)-\varphi_{\beta}(x)\right| \sigma(x)<k_{n} \quad\left(\alpha, \beta \geq \alpha_{0}\right) .
$$

Taking the limit over $\beta$ we get

$$
\sum_{x \in F_{n}}\left|\varphi_{\alpha_{0}}(x)-\varphi(x)\right| \sigma(x)<k_{n} \quad \text { for all } n \geq 1,
$$

and so

$$
\sum_{x \in F_{n}}\left|\varphi_{\alpha_{0}}(x)\right| \sigma(x) \geq k_{n}
$$


which contradicts the fact that $\varphi_{0}$ is in $\ell^{1}(\Sigma, \sigma)$. Hence $\varphi \in \ell^{1}(\Sigma, \sigma)$. Since $\beta^{1}(\Sigma, \sigma)$ has a base at zero consisting of a pointwise closed set, it follows easily that $\left(\varphi_{\alpha}\right)$ converges to $\varphi$ in the strict topology.

We denote the topology of pointwise convergence on $\ell^{1}(\Sigma, \sigma)$ by $\pi(\Sigma, \sigma)$.

Proposition 4.2. Let $\Sigma$ be a set and $\sigma$ be a positive function on $\Sigma$. On $\ell^{1}(\Sigma, \sigma)$ the topology $\pi(\Sigma, \sigma)$ coincides with the topology $\beta^{1}(\Sigma, \sigma)$ if and only if $\Sigma$ is finite.

Proof. Suppose that $\Sigma$ is infinite and let $A$ be an infinite countable subset of $\Sigma$, say

$$
A=\left\{x_{1}, x_{2}, \ldots\right\},
$$

such that $x_{i} \neq x_{j}$ for $i \neq j$. Then $A \backslash F \neq \emptyset$ for all finite subsets $F$ of $\Sigma$. Let $F$ be a finite subset of $\Sigma$ and choose $x_{F} \in A \backslash F$. For each natural number $n$, define the function $\varphi_{(F, n)} \in \ell^{1}(\Sigma, \sigma)$ by

$$
\varphi_{(F, n)}\left(x_{F}\right)=n ! \sigma^{-1}\left(x_{F}\right)
$$

and zero otherwise. Consider the set

$$
\Gamma=\{(F, n): F \subset \Sigma \text { is finite and } n \geq 1\}
$$

directed by $(F, n) \leq\left(F^{\prime}, n^{\prime}\right)$ if and only if $F \subset F^{\prime}$ and $n \leq n^{\prime}$. Then $\left(\varphi_{\gamma}\right)_{\gamma \in \Gamma}$ converges to zero in the $\pi(\Sigma, \sigma)$-topology.

Define $F_{n}:=\left\{x_{1}, x_{2}, \ldots, x_{n}\right\}$ and $r_{n}:=n !$. For any $\gamma:=(F, n) \in \Gamma$, the chosen $x_{F}$ is an element of $F_{n_{0}}$ for some $n_{0} \geq n$ and hence

$$
\sup \left\{\frac{1}{r_{n}} \sum_{x \in F_{n}}\left|\varphi_{\left(F, n_{0}\right)}(x)\right| \sigma(x): n \geq 1\right\} \geq 1 .
$$

In other words, $\mathcal{P}_{U}\left(\varphi_{\left(F, n_{0}\right)}\right) \geq 1$, where $U:=U\left(\left(F_{n}\right),\left(r_{n}\right)\right)$. Therefore $\left(\varphi_{\gamma}\right)_{\gamma \in \Gamma}$ could not converge to zero in the $\beta^{1}(\Sigma, \sigma)$-topology.

Proposition 4.3. Let $\Sigma$ be a set and $\sigma$ be a positive function on $\Sigma$. The topologies $\pi(\Sigma, \sigma)$ and $\beta^{1}(\Sigma, \sigma)$ coincide on all norm bounded subsets of $\ell^{1}(\Sigma, \sigma)$.

PROOF. We only need to prove that if $\left(\varphi_{\alpha}\right)$ is uniformly bounded and $\varphi_{\alpha} \longrightarrow 0$ in the $\pi(\Sigma, \sigma)$-topology, then $\varphi_{\alpha} \longrightarrow 0$ in the $\beta^{1}(\Sigma, \sigma)$-topology. Assume that $\left\|\varphi_{\alpha}\right\|_{1, \sigma} \leq M$ for all $\alpha$, and let $\left(\left(F_{n}\right),\left(r_{n}\right)\right) \in \mathcal{F} \times \mathcal{R}$. Let $\varepsilon>0$ and $n_{0} \geq 1$ be such that $\varepsilon r_{n_{0}} \geq M$. Then

$$
\sup \left\{\frac{1}{r_{n}} \sum_{x \in F_{n}}\left|\varphi_{\alpha}(x)\right| \sigma(x): n \geq n_{0}\right\} \leq \varepsilon .
$$

Since $\varphi_{\alpha} \longrightarrow 0$ in the $\pi(\Sigma, \sigma)$-topology, there exists $\alpha_{0}$ such that

$$
\sum_{x \in F_{n}}\left|\varphi_{\alpha}(x)\right| \sigma(x)<\varepsilon r_{n}
$$

for all $n<n_{0}$ and $\alpha \geq \alpha_{0}$. So, $\mathcal{P}_{U}\left(\varphi_{\alpha}\right) \leq \varepsilon$ for all $\alpha \geq \alpha_{0}$ and $U \in \mathcal{U}$. The result now follows. 
Proposition 4.4. Let $\Sigma$ be a set and $\sigma$ be a positive function on $\Sigma$. A sequence $\left(\varphi_{n}\right)$ in $\ell^{1}(\Sigma, \sigma)$ is $\beta^{1}(\Sigma, \sigma)$-convergent if and only if it is $\pi(\Sigma, \sigma)$-convergent and norm bounded.

Proof. The 'if' part follows from Proposition 4.3. To prove the converse, suppose that $\left(\varphi_{n}\right)$ is a sequence in $\ell^{1}(\Sigma, \sigma)$ which is not norm bounded. We show that $\left(\varphi_{n}\right)$ does not also converge in the strict topology. We can assume that $\left\|\varphi_{n}\right\|_{1, \sigma}>2^{n}$ for all $n \geq 1$. Select $K_{n}:=\left\{x_{1}, x_{2}, \ldots, x_{n}\right\}$ such that

$$
\sum_{x \in F_{n}}\left|\varphi_{n}(x)\right| \sigma(x) \geq 2^{n} .
$$

Setting $F_{n}:=\bigcup_{i=1}^{n} K_{i}$ and $r_{n}:=n$,

$$
\begin{aligned}
\mathcal{P}_{U}\left(\varphi_{n}\right) & :=\sup \left\{\frac{1}{r_{n}} \sum_{x \in F_{n}}\left|\varphi_{n}(x)\right| \sigma(x): n \geq 1\right\} \\
& \geq \sup \left\{\frac{2^{n}}{n}: n \geq 1\right\},
\end{aligned}
$$

where $U:=U\left(\left(F_{n}\right),\left(r_{n}\right)\right)$. So, $\left(\varphi_{n}\right)$ does not converge in the strict topology.

Let us recall some definitions from the theory of locally convex spaces. A locally convex space $(E, \tau)$ is called a barrelled space if each barrel set (that is, a closed convex balanced absorbing set) in $E$ is a neighbourhood of zero; it is called a bornological space when every convex balanced subset that absorbs bounded subsets in $E$ is a neighbourhood of zero.

Proposition 4.5. Let $\Sigma$ be a set and $\sigma$ be a positive function on $\Sigma$. Let $\tau$ be a locally convex topology such that $\sigma_{0}(\Sigma, \sigma) \leq \tau \leq \beta^{1}(\Sigma, \sigma)$. Then the following statements are equivalent.

(a) $\left(\ell^{1}(\Sigma, \sigma), \tau\right)$ is bornological.

(b) $\left(\ell^{1}(\Sigma, \sigma), \tau\right)$ is barrelled.

(c) $\left(\ell^{1}(\Sigma, \sigma), \tau\right)$ is quasi-barrelled.

(d) $\left(\ell^{1}(\Sigma, \sigma), \tau\right)$ is reflexive.

(e) $\left(\ell^{1}(\Sigma, \sigma), \tau\right)$ is metrizable.

(f) $\Sigma$ is finite.

PROOF. We only need to show that (f) holds if (a) or (b) holds. This follows from the fact that any metrizable space is a bornological space, and any reflexive space is a quasi-barrelled and therefore barrelled space.

First, suppose that (a) holds and let $I$ be the identity map from $\left(\ell^{1}(\Sigma, \sigma), \beta^{1}(\Sigma, \sigma)\right)$ into $\left(\ell^{1}(\Sigma, \sigma), n(\Sigma, \sigma)\right)$. Then $I$ is a bounded map by Proposition 2.1. Since by assumption $\left(\ell^{1}(\Sigma, \sigma), \beta^{1}(\Sigma, \sigma)\right)$ is a bornological space, $I$ is continuous. Therefore $n(\Sigma, \sigma)=\beta^{1}(\Sigma, \sigma)$. This, together with Proposition 2.2, implies (f).

Next, suppose that (b) holds. Then the unit ball

$$
\left\{\varphi \in \ell^{1}(\Sigma, \sigma):\|\varphi\|_{1, \sigma} \leq 1\right\}
$$


is a $\beta^{1}(\Sigma, \sigma)$-closed barrel set in $\ell^{1}(\Sigma, \sigma)$, and by assumption it is a $\beta^{1}(\Sigma, \sigma)$ neighbourhood of zero. That is, $n(\Sigma, \sigma) \leq \beta^{1}(\Sigma, \sigma)$. Invoke Proposition 2.2 to infer that $\Sigma$ is finite.

Let us recall that the locally convex space $(E, \tau)$ is said to be a dual space if there exists a locally convex space $\left(E_{0}, \tau_{0}\right)$ such that $(E, \tau)$ coincides with the strong dual of $\left(E_{0}, \tau_{0}\right)$.

Proposition 4.6. Let $\Sigma$ be a set and $\sigma$ be a positive function on $\Sigma$. The space $\left(\ell^{1}(\Sigma, \sigma), \beta^{1}(\Sigma, \sigma)\right)$ is a dual space if and only if $\Sigma$ is finite.

PROOF. We only prove the 'only if' part. By Theorem 3.1,

$$
\left(\ell^{1}(\Sigma, \sigma), \beta^{1}(\Sigma, \sigma)\right)^{*}=c_{0}(\Sigma, 1 / \sigma) .
$$

So, if $\left(\ell^{1}(\Sigma, \sigma), \beta^{1}(\Sigma, \sigma)\right)$ is a dual space, then $\left(\ell^{1}(\Sigma, \sigma), \beta^{1}(\Sigma, \sigma)\right)$ must be normable; this follows from the fact that a dual space whose dual is normable, itself is normable; see [5, Lemma 3.2]. Hence $\Sigma$ is finite.

Proposition 4.7. Let $\Sigma$ be a set, $\sigma$ be a positive function on $\Sigma$ and $A$ be a subset of $c_{0}(\Sigma, 1 / \sigma)$. Then the following statements are equivalent.

(a) $A$ is $\beta^{1}(\Sigma, \sigma)$-equicontinuous.

(b) $A$ is $\|\cdot\|_{\infty, \sigma}$-bounded and, for $\varepsilon>0$, there exists a finite subset $F$ of $\Sigma$ such that $\langle|f|,|\varphi|\rangle<\varepsilon$ for all $f \in A$ and $\varphi \in \ell^{1}(\sigma, \Sigma)$ with $\|\varphi\|_{1, \sigma} \leq 1$ and $\operatorname{coz}(\varphi) \subset \Sigma \backslash F$.

(c) A is $\|\cdot\|_{\infty, \sigma}$-bounded and, for $\varepsilon>0$, there exists a finite subset $F$ of $\Sigma$ such that $|f(x)|<\varepsilon$ for all $f \in A$ and $x \in \Sigma \backslash F$.

Proof. (a) $\Rightarrow$ (b). Norm boundedness of $A$ follows easily from definition and $\beta^{1}(\Sigma, \sigma)$-boundedness of the unit ball of $\ell^{1}(\Sigma, \sigma)$. Now, choose a neighbourhood $U\left(\left(F_{n}\right),\left(r_{n}\right)\right)$ such that

$$
|\langle f, \varphi\rangle| \leq 1
$$

for $f \in A$ and $\varphi \in U\left(\left(F_{n}\right),\left(r_{n}\right)\right)$. For an arbitrary $\varepsilon>0$, choose $n_{0} \in \mathbb{N}$ such that $\varepsilon r_{n_{0}}>1$. Set

$$
F:=\bigcup_{n=1}^{n_{0}} F_{n} .
$$

We then have $\varphi \in \varepsilon U\left(\left(F_{n}\right),\left(r_{n}\right)\right)$ for all $\varphi \in \ell^{1}(\Sigma, \sigma)$ with $\|\varphi\|_{1, \sigma} \leq 1$ and

$$
\operatorname{coz}(\varphi) \subseteq \Sigma \backslash F
$$

So $|\langle f, \varphi\rangle| \leq \varepsilon$ for $f \in A$. This implies that $\langle|f|,|\varphi|\rangle \leq \varepsilon$.

(b) $\Rightarrow$ (c). Let $\varepsilon>0$ and $F$ be as in part (b) and note that, for any point $x \in \Sigma \backslash F$ and $f \in A$,

$$
|f(x)|=\left\langle|f|, \delta_{x}\right\rangle<\varepsilon .
$$


(c) $\Rightarrow$ (a). For $n \in \mathbb{N}$, choose a finite set $F_{n}$ such that $|f(x)| \leq 2^{-2 n}$ for $x \in \Sigma \backslash F_{n}$. Setting $r_{n}:=2^{n}$ and $U:=U\left(\left(F_{n}\right),\left(r_{n}\right)\right)$, for each $f \in A$,

$$
\begin{aligned}
|\langle f, \varphi\rangle| & =\sum_{x \in F_{1}}|f(x) \| \varphi(x)|+\sum_{n=1}^{\infty}\left(\sum_{x \in F_{n+1} \backslash F_{n}}|f(x) \| \varphi(x)|\right) \\
& \leq \sum_{x \in F_{1}}|f(x) \| \varphi(x)|+\sum_{n=1}^{\infty}\left(2^{-2 n} \sum_{x \in F_{n+1} \backslash F_{n}}|\varphi(x)|\right) \\
& \leq \sum_{x \in F_{1}}|f(x) \| \varphi(x)|+\sum_{n=1}^{\infty} 2^{-2 n}\left(\begin{array}{c}
n+1 \\
2
\end{array}\right) \\
& \leq 2\|f\|_{\infty, \sigma}+2,
\end{aligned}
$$

for all $\varphi \in U$ and $f \in A$. So, $f$ is bounded on $U$ for all $f \in A$. This completes the proof.

A locally convex space $(E, \tau)$ is called a Mackey space if $\tau$ coincides with the Mackey topology $\mu\left(E, E^{*}\right)$; also $(E, \tau)$ is called a DF space if $E$ possesses a fundamental sequence of bounded sets (that is, a sequence of bounded sets $\left(B_{n}\right)$ such that $B_{n}+B_{n} \subset B_{n+1}$ ), and if every strongly bounded countable union of equicontinuous subsets of $E^{*}$ is equicontinuous; see [6] for more details.

Proposition 4.8. Let $\Sigma$ be a set and $\sigma$ be a positive function on $\Sigma$. Then the following statements are equivalent.

(a) $\left(\ell^{1}(\Sigma, \sigma), \beta^{1}(\Sigma, \sigma)\right)$ is Mackey space.

(b) $\left(\ell^{1}(\Sigma, \sigma), \beta^{1}(\Sigma, \sigma)\right)$ is DF space.

(c) $\Sigma$ is finite.

Proof. (a) $\Rightarrow$ (c). Let $\Delta=\left\{\delta_{x}: x \in \Sigma\right\} \subseteq c_{0}(\Sigma, 1 / \sigma)$. Then an easy application of the Smulian-Eberlein and Krein theorems implies weak compactness of $\Delta$ and its closed convex hull. It follows from [15, Theorem 9.4.2] that $\Delta$ is equicontinuous. Now invoke Proposition 4.7 to conclude that $\Sigma$ is finite.

(b) $\Rightarrow$ (c). If there is a sequence $\left(x_{n}\right)$ of distinct elements of $\Sigma$, then $\bigcup_{n=1}^{\infty}\left\{\delta_{x_{n}}\right\}$ is equicontinuous by (b). So, $\bigcup_{n=1}^{\infty}\left\{\delta_{x_{n}}\right\}$ satisfies condition (b) in Proposition 4.7, a contradiction.

Proposition 4.9. Let $\Sigma$ be a set and $\sigma$ be a positive function on $\Sigma$. The strict topology is the finest locally convex topology that agrees with the strict topology on norm bounded subsets of $\ell^{1}(\Sigma, \sigma)$ if and only if $\Sigma$ is countable.

PROOF. Let $\beta^{0}(\Sigma, \sigma)$ denote the locally convex topology generated by seminorms

$$
\mathcal{P}_{f}(\varphi)=\|f \varphi\|_{1},
$$


where $f \in c_{0}(\Sigma, 1 / \sigma)$. By [10], $\beta^{0}(\Sigma, \sigma)$ is the finest locally convex topology on $\ell^{1}(\Sigma, \sigma)$ that agrees with $\beta^{0}(\Sigma, \sigma)$ on bounded sets. It is clear that $\beta^{1}(\Sigma, \sigma) \leq$ $\beta^{0}(\Sigma, \sigma)$. Suppose that $\Sigma$ is uncountable. Let $V_{f}$ be the $\beta^{0}(\Sigma, \sigma)$-neighbourhood

$$
\left\{\varphi \in \ell^{1}(\Sigma, \sigma):\|f \varphi\|_{1} \leq 1\right\}
$$

of zero, where $f \in c_{0}(\Sigma, 1 / \sigma)$ and $f(x) \neq 0$ for all $x \in \Sigma$.

Then $V_{f}$ is not a $\beta^{1}(\Sigma, \sigma)$-neighbourhood; indeed, if there exists $\left(\left(F_{n}\right),\left(r_{n}\right)\right) \in$ $\mathcal{F} \times \mathcal{R}$ such that $U\left(\left(F_{n}\right),\left(r_{n}\right)\right) \subseteq V_{f}$, then $r_{n} \delta_{x} \in U\left(\left(F_{n}\right),\left(r_{n}\right)\right)$ for all $n \geq 1$ and $x \in F_{n}$, but $r_{n} \delta_{x} \notin V_{f}$ for some $n \geq 1$ and $x \in F_{n}$.

Conversely, let $\Sigma$ be countable and

$$
V_{f}=\left\{\varphi \in \ell^{1}(\Sigma, \sigma):\|f \varphi\|_{1} \leq 1\right\} .
$$

For $n \in \mathbb{N}$, choose a finite set $F_{n} \subset \Sigma$ such that

$$
n 2^{n} f(x)<\sigma(x)
$$

for all $x \in \Sigma \backslash F_{n}$. We show that

$$
U\left(\left(F_{n}\right),(n)\right) \subseteq V_{f} .
$$

Let $\varphi \in U\left(\left(F_{n}\right),(n)\right)$. Then

$$
\begin{aligned}
\sum_{x \in F_{n}}|\varphi(x) f(x)| & =\sum_{m=1}^{n}\left(\sum_{x \in F_{m} \backslash F_{m-1}}|f(x) \varphi(x)|\right) \\
& \leq \sum_{m=1}^{n}\left(\sum_{x \in F_{m} \backslash F_{m-1}} \frac{1}{m 2^{m}}|\varphi(x)| \sigma(x)\right) \\
& \leq \sum_{m=1}^{n} \frac{1}{2^{m}} .
\end{aligned}
$$

Since $f(x)=0$ for all $x \in \Sigma \backslash \bigcup_{n=1}^{\infty} F_{n}$, it follows that $\|f \varphi\|_{1} \leq 1$ as required.

A locally convex space $E$ is called quasi-normable if every open subset $U \subseteq E$ contains an open subset $V \subseteq E$ such that, for each $\alpha>0$, we can find a bounded subset $B \subseteq E$ with $V \subseteq B+\alpha U$.

PROPOSITION 4.10. Let $\Sigma$ be a set and $\sigma$ be a positive function on $\Sigma$. Then $\ell^{1}(\Sigma, \sigma)$ with the strict topology is always quasi-normable.

PROOF. Let $U=U\left(\left(F_{n}\right),\left(r_{n}\right)\right)$ be an arbitrary $\beta^{1}(\Sigma, \sigma)$-neighbourhood of zero. Choose a sequence of positive numbers $\left(s_{n}\right) \in \mathcal{R}$ such that $s_{n} \leq r_{n}$ and $\left(s_{n} / r_{n}\right)$ tends to zero. Define

$$
V=V\left(\left(F_{n}\right),\left(s_{n}\right)\right) .
$$

For a given $0<\alpha<1$, choose a natural number $n_{0}$ such that $s_{n} \leq \alpha r_{n}$ for all $n \geq n_{0}$. It is easy to see that each $\varphi \in V$ is the sum of two functions $\chi_{F_{n_{0}}} \varphi$ and $\left(1-\chi_{F_{n_{0}}}\right) \varphi$ 
such that

$$
\chi_{F_{n_{0}}} \varphi \in\left\{\varphi \in \ell^{1}(\Sigma, \sigma):\|\varphi\|_{1, \sigma} \leq s_{n_{0}}\right\}
$$

and $\left(1-\chi_{F_{n_{0}}}\right) \varphi \in \alpha U$, and the proof is complete.

Let $E$ be a locally convex space, and let $\mathcal{U}$ be a base at zero for $E$ consisting of absolutely convex sets. The linear space of all sequences $\left(x_{n}\right)$ in $E$ such that $\left(\left\langle f, x_{n}\right\rangle\right)_{n} \in \ell^{1}(\mathbb{N})$ for all $f \in E^{*}$ is denoted by $\ell_{1}[E]$. The seminorms

$$
\varepsilon_{U}\left(\left(x_{n}\right)\right):=\sup \left\{\sum_{n=1}^{\infty}\left|\left\langle f, x_{n}\right\rangle\right|: f \in U^{\circ}\right\} \quad(U \in \mathcal{U})
$$

generate a locally convex topology on $\ell_{1}[E]$. A sequence $\left(x_{n}\right)$ in $E$ is called absolutely Cauchy if

$$
\pi_{U}\left(\left(x_{n}\right)\right):=\sum_{n=1}^{\infty} q_{U}\left(x_{n}\right)<\infty
$$

for all $U \in \mathcal{U}$, where $q_{U}$ denotes the Minkowski functional of $U$. The linear space of all absolutely Cauchy sequences in $E$ is denoted by $\ell_{1}\{E\}$ equipped with the topology given by the seminorms $\pi_{U}$. A locally convex space $E$ is called nuclear if $\ell_{1}\{E\}=\ell_{1}[E]$ topologically and algebraically; for more details, see [6], for example.

The following theorem shows that $\left(\ell^{1}(\Sigma, \sigma), \beta^{1}(\Sigma, \sigma)\right)$ behaves as a Banach space with respect to nuclearity.

PROPOSITION 4.11. Let $\Sigma$ be a set and $\sigma$ be a positive function on $\Sigma$. Then $\ell^{1}(\Sigma, \sigma)$ with strict topology is a nuclear space if and only if $\Sigma$ is finite.

ProOF. Proposition 4.7 implies that $\left(\ell^{1}(\Sigma, \sigma), \beta^{1}(\Sigma, \sigma)\right)$ is sequentially evaluable; recall that a locally convex space $(E, \tau)$ is said to be sequentially evaluable if every $\beta\left(E^{*}, E\right)$-convergent sequence in $E^{*}$ is equicontinuous. Note also that $\ell^{1}(\Sigma, \sigma)$ has a fundamental sequence of bounded sets (take, for example,

$$
B_{n}=\left\{\varphi:\|\varphi\|_{1, \sigma} \leq n\right\}
$$

for all $n \geq 1)$. Now, if $\left(\ell^{1}(\Sigma, \sigma), \beta^{1}(\Sigma, \sigma)\right)$ is nuclear, then [9, Theorem 2.14] implies that the Banach space

$$
\left(\ell^{1}(\Sigma, \sigma), \beta^{1}(\Sigma, \sigma)\right)^{*}=c_{0}(\Sigma, 1 / \sigma)
$$

is nuclear, and hence $\Sigma$ must be finite.

\section{An application to semigroup algebra}

Let $S$ be a semigroup and $\sigma$ be a weight function on it; that is, a positive function with $\sigma(s t) \leq \sigma(s) \sigma(t)$ for all $s, t \in S$. The convolution product on $\ell^{1}(S, \sigma)$ is defined by

$$
(\varphi * \psi)(x)=\sum_{s t=x} \varphi(s) \psi(t)
$$


for $\varphi, \psi \in \ell^{1}(S, \sigma)$ and $x \in S$ when $s t=x$ has a solution, and $(\varphi * \psi)(x)=0$ otherwise.

Here, we consider the semigroup algebra $\ell^{1}(S, \sigma)$ with convolution as multiplication, and prove separate $\beta^{1}(S, \sigma)$-continuity of this multiplication for a large class of semigroups.

First, let us recall that a semigroup $S$ is called finitely cancellative if

$$
t^{-1} x=\{s \in S: t s=x\}
$$

is finite for all $x, t \in S$.

Proposition 5.1. Suppose that $S$ is a countable finitely cancellative semigroup. Then $\left(\ell^{1}(S, \sigma), \beta^{1}(S, \sigma)\right)$ with convolution as multiplication is a complete semitopological algebra.

Proof. Since $S$ is countable, in view of Proposition 4.9, we only need to show that convolution on $\left(\ell^{1}(S, \sigma), \beta^{1}(S, \sigma)\right)$ is $\beta^{1}(S, \sigma)$-continuous on $\beta(\Sigma, \sigma)$-bounded sets; see [6]. Let $\left(\varphi_{\alpha}\right)$ be a norm bounded net in $\ell^{1}(S, \sigma)$ convergent to zero in $\beta^{1}(S, \sigma)$. Let $\psi \in \ell^{1}(S, \sigma)$ and fix $x_{0} \in S$. Choose a finite set $F \subseteq S$ such that

$$
\sum_{t \in S \backslash F}|\psi(t)| \sigma(t)<\frac{\varepsilon \sigma\left(x_{0}\right)}{2 M},
$$

where $M$ is a bound for the net $\left(\varphi_{\alpha}\right)$. Then $F^{-1} x_{0}$ is finite by the finite cancellativity of $S$. So, if we put

$$
F_{n}:=F^{-1} x_{0} \quad \text { and } \quad r_{n}:=\frac{\varepsilon n \sigma\left(x_{0}\right)}{2\|\psi\|_{1, \sigma}},
$$

then $\left(\left(F_{n}\right),\left(r_{n}\right)\right) \in \mathcal{F} \times \mathcal{R}$, and so there is $\alpha_{0}$ such that $\varphi_{\alpha} \in U\left(\left(F_{n}\right),\left(r_{n}\right)\right)$ for all $\alpha \geq \alpha_{0}$. In particular,

$$
\sum_{s \in F^{-1} x_{0}}\left|\varphi_{\alpha}(s)\right| \sigma(s)<\frac{\varepsilon \sigma\left(x_{0}\right)}{2\|\psi\|_{1, \sigma}}
$$

for all $\alpha \geq \alpha_{0}$, where

$$
F^{-1} x_{0}:=\left\{s \in S: t s=x_{0} \text { for some } t \in F\right\} .
$$

Now, for each $\alpha \geq \alpha_{0}$,

$$
\begin{aligned}
\left|\sum_{s t=x_{0}} \varphi_{\alpha}(s) \psi(t)\right| \leq & \sigma\left(x_{0}\right)^{-1} \sum_{s t=x_{0}}\left|\varphi_{\alpha}(s)\right||\psi(t)| \sigma(s) \sigma(t) \\
\leq & \sigma\left(x_{0}\right)^{-1} \sum_{t \in F} \sum_{s \in F^{-1} x_{0}}\left|\varphi_{\alpha}(s)\right| \sigma(s)|\psi(t)| \sigma(t) \\
& \quad+\sigma\left(x_{0}\right)^{-1} \sum_{t \in S \backslash F} \sum_{s \in(S \backslash F)^{-1} x_{0}}\left|\varphi_{\alpha}(s)\right| \sigma(s)|\psi(t)| \sigma(t)
\end{aligned}
$$




$$
\begin{aligned}
& \leq \sigma\left(x_{0}\right)^{-1}\|\psi\|_{1, \sigma} \sum_{s \in F^{-1} x_{0}}\left|\varphi_{\alpha}(s)\right| \sigma(s) \\
& \quad+\sigma\left(x_{0}\right)^{-1} M \sum_{t \in S \backslash F}|\psi(t)| \sigma(t) \\
& \leq \varepsilon
\end{aligned}
$$

Hence, $\left(\varphi_{\alpha} * \psi\right)\left(x_{0}\right) \longrightarrow 0$ and $\varphi_{\alpha} * \psi \longrightarrow 0$ in the $\beta^{1}(S, \sigma)$-topology.

The following example shows that Proposition 5.1 does not hold in general.

EXAmple 5.2. Let $S=\{0\} \cup\{1 / n: n \in \mathbb{N}\}$. Then $S$ with the operation $s t=$ $\max \{s, t\}$ is a countable semigroup with identity. It is easy to see that $\varphi \mapsto \varphi * \delta_{1}$ is not $\beta^{1}(S, \sigma)$-continuous on $\ell^{1}(S, 1)$; in particular, $S$ is not finitely cancellative.

In conclusion, we give a special case of Proposition 5.1 which partially answers a question raised by Singh in [12].

COROLlary 5.3. If $G$ is a countable group, then $\left(\ell^{1}(G), \beta^{1}(G)\right)$ with convolution as multiplication is a complete semi-topological algebra.

\section{Acknowledgements}

The research of the first named author was supported by the Research Institute for Fundamental Science, Tabriz, Iran. The second named author thanks the Center of Excellence for Mathematics at the Isfahan University of Technology.

\section{References}

[1] R. C. Buck, 'Bounded continuous functions on a locally compact space', Michigan Math. J. 5 (1958), 95-104.

[2] H. S. Collins, 'Strict, weighted, and mixed topologies and applications', Adv. Math. 19 (1976), 207-237.

[3] J. R. Dorroh, 'The localization of the strict topology via bounded sets', Proc. Amer. Math. Soc. 20 (1969), 413-414.

[4] J. Ferrera and A. Prieto, 'The strict topology on spaces of bounded holomorphic functions', Bull. Aust. Math. Soc. 49 (1994), 249-256.

[5] D. Gulick, 'Duality theory for the strict topology', Studia Math. 49 (1974), 195-208.

[6] H. Jarchow, Locally Convex Spaces (B. G. Teubner, Stuttgart, 1981).

[7] L. A. Khan, The General Strict Topology on Topological Modules, Contemporary Mathematics, 435 (American Mathematical Society, Providence, RI, 2007), pp. 253-263.

[8] J. M. Robertson and H. C. Wiser, 'A note on polar topologies', Canad. Math. Bull. 11 (1968), 607-609.

[9] W. Ruess, 'On the locally convex structure of strict topologies', Math. Z. 153 (1977), 179-192.

[10] F. Sentilles and D. Taylor, 'Factorization in Banach algebras and the general strict topology', Trans. Amer. Math. Soc. 142 (1969), 141-152.

[11] J. H. Shapiro, 'The bounded weak star topology and the general strict topology', J. Funct. Anal. 8 (1971), 275-286.

[12] A. I. Singh, ' $L_{0}^{\infty}(G)^{*}$ as the second dual of the group algebra $L^{1}(G)$ with a locally convex topology', Michigan Math. J. 46 (1999), 143-150. 
[13] C. Swartz, An Introduction to Functional Analysis, Pure and Applied Mathematics, 157 (Marcel Dekker, New York, 1992).

[14] R. F. Wheeler, 'A survey of Baire measures and strict topologies', Expo. Math. 1 (1983), 97-190.

[15] A. Wilansky, Modern Methods in Topological Vector Spaces (McGraw-Hill, New York, 1978).

[16] J. Zafarani, 'A space of vector-valued measures and a strict topology', Manuscripta Math. 39 (1982), 147-153.

SAEID MAGHSOUDI, Department of Mathematics, Zanjan University, Zanjan, 313, Iran

and

Research Institute for Fundamental Science, Tabriz, Iran

e-mail: s_maghsodi@znu.ac.ir

RASOUL NASR-ISFAHANI, Department of Mathematical Sciences, Isfahan University of Technology, Isfahan, 84156-83111, Iran

e-mail: isfahani@cc.iut.ac.ir 